\title{
Recovery of function after brain damage: Toward understanding the deficit
}

\author{
T. E. LeVERE, N. DAVIS, and L. GONDER \\ Neuropsychology Laboratory, North Carolina State University, Raleigh, North Carolina 27650
}

\begin{abstract}
The present research was based on the proposition that understanding the process of recovery of function after brain injury is ultimately determined by defining the nature of the behavioral dysfunction. For example, the disruption of a learned brightness discrimination by visual decortication may reflect a loss of preoperative memories, the motivation to perform, or the ability to utilize available neural mechanisms. Depending upon which of these represents the behavioral deficit determines what controls recovery, simply because recovery would, respectively, involve relearning processes, motivational processes, or memory processes. Since we have previously demonstrated that the behavioral deficit is not a function of the loss of preoperatively established memory traces, the present research questioned whether the behavioral deficit might be characterized as: (1) a dysfunction in the motivation to perform or (2) a dysfunction in the ability to utilize what neural mechanisms were spared by the brain injury. Both of the experiments employed a proactive design strategy to test whether what was previously learned would affect subsequent behavior in the brain-damaged rat. In both experiments, such proactive effects were observed. Thus, the behavioral dysfunction following striate ablation is not due to a loss of memory, a failure of motivation, or a lack of utilization. On the basis of these data, it is then suggested that perhaps the behavioral deficit following sensory neocortical lesions might be best understood in view of transfer of training.
\end{abstract}

The belief that some amount of recovery of function will follow brain injury is for all intents axiomatic. This is particularly true with respect to the disruption of learned behaviors following neocortical insult, and considerable research has been devoted to understanding the controlling variables. From this effort, we have discovered that brain injury sustained early in life is less disruptive than brain injury sustained in adulthood (Akert, Orth, Harlow, \& Schlitz, 1960; Benjamin \& Thompson, 1959; Kennard, 1936, 1938, 1942). But not always (Isaacson, 1975; Johnson \& Almli, 1978). And we have discovered that overtraining will protect learned behaviors against the disruptive effects of brain injury (Chow \& Survis, 1958; Lukaszewska \& Thompson, 1967; Orbach \& Fantz, 1958; Thatcher \& Kimble, 1966; Weese, Neimand, \& Finger, 1973). But not always (Glendenning, 1972; Lashley, 1921; Rabb \& Ades, 1946). And we have discovered that seriatim brain injury is less debilitating than comparable single stage insult (see review by Finger, 1978). But not always (Dawson, Conrad, \& Lynch, 1973; Issacson \& Schmaltz, 1968; Kircher, Braun, Meyer, \& Meyer,

This research was supported by Research Grant NS-12459 from the National Institute of Neurological and Communicative Disorders and Stroke, U.S. Department of Health, Education and Welfare, to T. E. LeVere. The authors wish to thank P. Knowles for her assistance in the preparation of this report. Requests for reprints should be directed to T. E. LeVere, Neuropsychology Laboratory, Department of Psychology, North Carolina State University, Raleigh, North Carolina 27650.
1970; LeVere, 1969; LeVere \& Weiss, 1973; Meyer, Hughes, Buchholz, Dalhouse, Enloe, \& Meyer, 1976). The ledger may, of course, be extended, but unfortunately the net result shows no improvement.

One reason for these equivocal conclusions concerning recovery of function is that much of the research has principally concentrated on the process - that is, recovery-and not the cause-that is, the behavioral dysfunction. Yet, the fundamental determinant of recovery of function is the nature of the behavioral dysfunction. To simply dismiss the behavioral dysfunction as a deficit or an inability to initially perform some even very specific behavior is, at best, a gross and oversimplified description of an outcome which may have any one of a number of causes. And if these causes are unspecified beyond some recognition of the inability to perform, then how can we expect anything but vagarious information concerning how the behavioral deficit may be reversed? For example, consider the disruption of a preoperatively learned brightness discrimination following striate ablation. The postoperative behavioral dysfunction might reflect the destruction of the neural mechanisms associated with the "memory engrams" or "memory traces" of the preoperatively learned behavior. Or the neural substrates of the memory traces might be spared and the behavioral deficit might reflect the destruction of the neural mechanisms associated with the motivation to perform the discrimination. Or the neural substrates of both memory and motivation might be spared and 
the behavioral deficit might reflect an inability to utilize what is spared. The variables influencing the process of recovery of function in each instance would be quite different simply because the controlling factors would be relearning, motivation and memory processes, respectively. Clearly, even to begin to appreciate the process of recovery of function, we necessarily must first specify what is being recovered, that is, the nature of the behavioral dysfunction.

Following this line of reasoning, we initially inquired as to whether the disruption of a preoperatively learned brightness discrimination by visual neodecortication represented a loss of memory traces, or memory engrams (LeVere \& Morlock, 1973, 1974). Our procedure was to test for sparing with a proactive interference paradigm. Specifically, we postoperatively retrained rats on the original preoperatively learned brightness discrimination or a brightness discrimination which reversed the reward contingencies of the preoperative problem. Our logic was that if the memory traces of the preoperative learning were destroyed by the visual lesion, then recovery of function should represent new learning. And if the memory traces did not exist and the lesioned rat was learning anew, then it should matter little what the brain-injured animal was learning. Accordingly, we predicted that if the behavioral dysfunction following visual decortication was a loss of memory, then both the reversal and nonreversal groups should relearn the brightness discrimination with equal efficiency. However, this prediction was not realized and the animals postoperatively trained on the reversal brightness discrimination were significantly impaired. Since this result could occur only if the visual lesion spared the memory traces of the preoperatively learned discrimination, we concluded that, in this case, the behavioral deficit must reflect something other than the destruction and loss of preoperative memories.

Since it is not possible to characterize the behavioral deficit produced by striate lesions as a loss of memory, the present research reports two experiments which tested whether a motivation and/or utilization dysfunction might better characterize the behavioral effects of visual decortication. While the experiments differ in detail, the underlying strategy is the same as that employed by LeVere and Morlock $(1973,1974)$. In each instance, the principal experimental question is whether the previous learning is able to influence subsequent behavior. If it does, then this is taken as evidence of sparing and support for the conclusion that the parameter under investigation is not at the root of the behavioral deficit suffered by the brain-lesioned animal.

\section{EXPERIMENT 1}

The importance of motivation and the possibility that the behavioral deficit may reflect a dysfunction associated with motivation is suggested by certain of our previous research. This research was concerned with the conditions necessary for spared memory traces to influence postoperative recovery of function (LeVere \& Davis, 1977). The major finding of this research was that if the same motivational conditions occurred during preoperative training and postoperative retraining, then the spared memory traces of the preoperatively learned behavior would influence recovery of function. However, if the postoperative motivation was different, then the spared memory traces would have little influence on recovery of function. Thus, it would appear that what motivates the individual may be critical to the postoperative expression of spared neural mechanisms. And, to take the argument a step further, might not this motivational dependency be indicative of a more general dysfunction? That is, the occurrence of an overt behavior is dependent not only on the neural mechanisms requisite to the behavior per se, but also on the neural mechanisms associated with the motivation to perform the behavior. And if preoperative memory traces are spared by striate lesions, then is it not possible that the behavioral deficit occurs because the brain lesion produces some dysfunction in the motivation to perform? The first experiment was an attempt to test this possibility.

Our strategy to determine whether or not striate lesions affect an individual's motivation to perform involved initially training different groups of rats on the same brightness discrimination but for different levels of incentive. Our prediction was that if the behavioral deficit represented a dysfunction of the neural mechanisms associated with motivation to perform, then the groups preoperatively trained under different incentive levels should show similar behavior during a postoperative retention test. On the other hand, if the neural mechanisms associated with the motivation to perform are spared by visual decortication, then the groups of animals preoperatively trained under different incentive levels should behave differently on a postoperative retention test.

\section{Method}

Subjects. The subjects for this experiment were 17 male hooded rats between the ages of 90 and 100 days. The animals were housed individually in wire mesh cages in a room with a reversed 10:14-h day:night cycle. Throughout the experiment, the subjects were allowed free access to food and water.

Apparatus. The training apparatus was our standard Y-maze previously described by LeVere and Morlock $(1973,1974)$. Basically, the maze is composed of a start compartment, a choice area, and two goal compartments which are distinguished by transilluminated entrance doors. The rear wall of each goal compartment is made of clear plastic so that flood lamps at the ends of the tunnels extending backwards from the rear of each goal compartment can illuminate the entrance doors. Each flood lamp was powered by the ac voltage necessary to produce a $63-\mathrm{fL}$ brightness on the translucent entrance of the goal compartment. This 63-fL brightness was the brighter discriminative cue; the dimmer discriminative cue was produced by interposing a small aperture in front of the other flood lamp to restrict its light output to 
$1 \mathrm{fL}$. The animal gained entrance to the correct goal area by knocking down the translucent door at its entrance. The translucent door to the incorrect goal area was always locked. Whenever the animal knocked down the door to the correct goal area, a switch was immediately tripped and both flood lamps were instantaneously extinguished. For the present procedures, the brighter 63-fL cue always signified the correct goal compartment.

The animals learned the brightness discrimination to avoid/ escape electric shock applied to the grid floor of the start compartment and choice area. During preoperative discrimination training, the apparatus was programmed to allow the animal $10 \mathrm{sec}$ to exit the start compartment before the occurrence of a pulsating ( $1 \mathrm{sec}$ on, $.5 \mathrm{sec}$ off) shock. After exiting the start compartment, the animal was allowed an additional 10 -sec shockfree period to execute a choice response. If the animal failed to respond within this 10 -sec period, or if it attempted to knock down the locked door to the incorrect goal compartment, the pulsating shock was reinstituted until a correct response was completed. Training progressed at the rate of 10 trials/day for 7 days each week until the animal attained a criterion of 9 first-choice correct responses within a single day's training.

The postoperative retention test was identical to preoperative training except that: (1) the animals were never shocked, and (2) the entrance doors to both the correct and the incorrect goal areas were unlocked.

Procedure. The 17 animals of this experiment were randomly assigned to three experimental groups. Each of these groups experienced different intensities of footshock during preoperative discrimination training. The three shock intensities were $.3, .5$, and $.8 \mathrm{~mA}$, according to the dial settings of our Grason-Stadler shock source. With the exception of different shock intensities, the training of each group followed the same paradigm. First, the animals were habituated and shaped to respond in the apparatus. During shaping, the entrances to the goal areas were illuminated with a brightness midway between the correct and incorrect cues of the brightness discrimination. Following shaping, which typically required 2 to 4 days, the rats were trained to the $9 / 10$ criterion on the brightness discrimination. Within $48 \mathrm{~h}$ following criterion performance, each animal was subjected to posterior neodecortication. After surgery, the animals were allowed a 2-week rest period, following which they were returned to the apparatus for a 1-day postoperative test procedure.

Our main interest during the postoperative retention test was twofold. First, would the animals respond (run from the start compartment, knock down the translucent door to a goal area, and enter the goal area) even though there was no physical necessity to do so? Second, assuming the animals responded, would the different intensities of footshock experienced by the experimental groups during preoperative training yield different postoperative responding? Accordingly, our postoperative dependent measure was response latency as measured from the time the guillotine door of the start compartment was raised to when the subject entered a goal area with all four feet. There was, however, an upper response latency limit of $120 \mathrm{sec}$, after which, if the subject had not entered the goal area, it was removed from the apparatus to await the next postoperative test trial.

Surgery was performed under clean procedures using sodium pentobarbital anesthesia administered IP. The lesion was intended to be quite extensive and include all neocortex posterior to a line beginning at bregma and becoming tangential to the rhinal sulcus. This purposely large lesion was to insure inclusion of all neocortical areas receiving visual thalamic projections as described by Hughes (1977). All animals received a postoperative injection of a broadband antibiotic as a prophylactic.

After each animal had finished its postoperative test, it was given a lethal dose of sodium pentobarbital and perfused according to procedures described by LeVere and Morlock $(1973,1974)$. The brains of the animals were then removed and the extent of the lesion drawn on surface diagrams. Following this, the brains were frozen and sectioned at $50 \mu$; every fourth section through the lesion area and the lateral geniculate nucleus was saved and stained with cresyl violet. These sections were then analyzed for a retrograde degeneration to determine the completeness of each lesion as well as whether or not any lesions invaded subcortical areas.

\section{Results and Discussion}

Histology. Figure 1 presents diagrams of the extent of the neocortical insult as well as the amount of cellular degeneration within the dorsal lateral geniculate nucleus. The upper portion of the figure represents the rat sustaining the smallest neocortical lesion (42.2\% of neocortex), while the lower portion represents the rat sustaining the largest lesion $(57.5 \%$ of neocortex). The average lesion size was $49.9 \%$, $47.6 \%$, and $47.9 \%$, respectively for the .3-, .5-, and .8-mA experimental groups. An analysis of variance indicated no significant differences in lesion size $(F<1)$.

Examination of the cresyl violet stained sections indicated complete destruction of neocortex through-
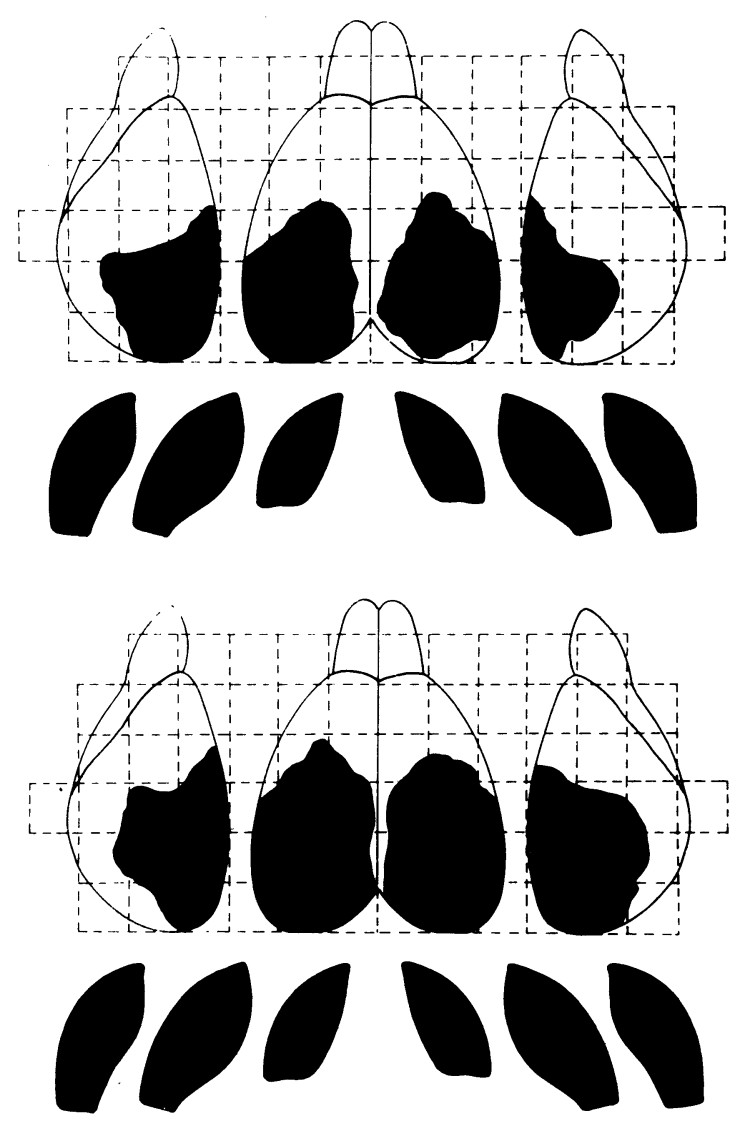

Figure 1. Neocortical destruction and retrograde degeneration within the dorsal lateral geniculate nucleus (black areas) for the rat receiving the smallest (top) and the rat receiving the largest (bottom) lesion. The frontal sections through the dorsal portion of the lateral geniculate nucleus correspond to approximately $2.5 \mathrm{~mm}$ (extreme left and right) $3.1 \mathrm{~mm}$ (second from left and right), and $3.7 \mathrm{~mm}$ (middle) from the interaural line. 


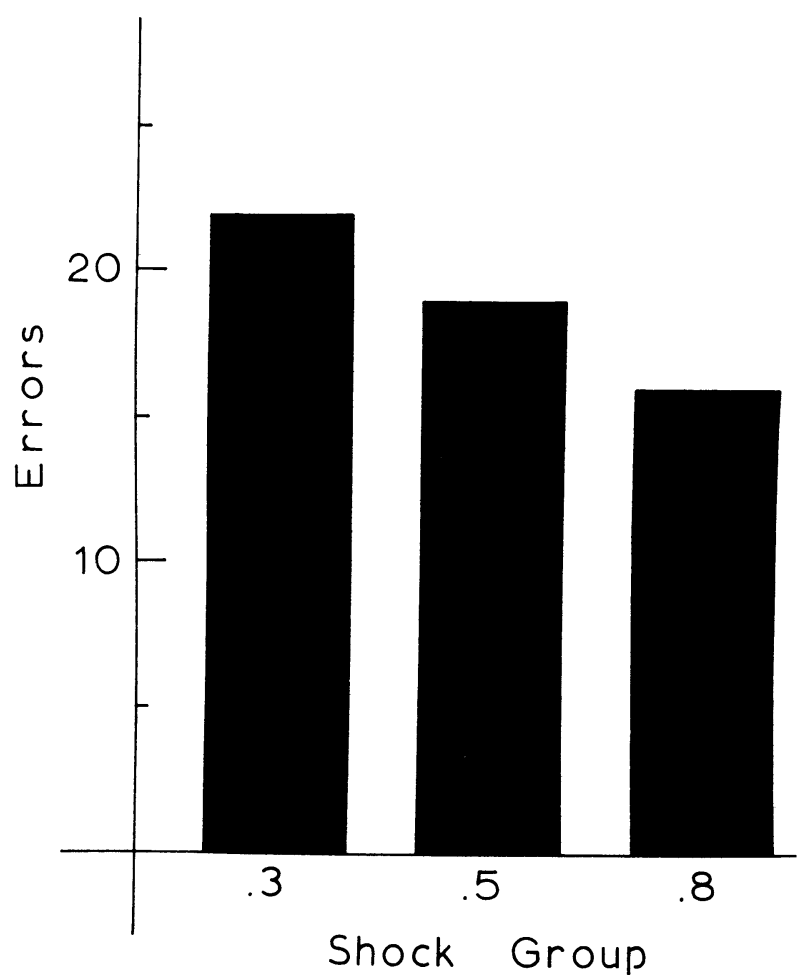

Figure 2. Mean errors to $9 / 10$ criterion for each experimental group.

out all six layers. While there was considerable degeneration of the underlying corpus callosum, the hippocampus and thalamic nuclei were never invaded. Within the dorsal lateral geniculate nucleus, there was, in all cases, complete degeneration. The lateral portion of the lateral posterior thalamic nucleus also indicated some degeneration at its border with the lateral geniculate nucleus. However, it was often difficult to distinguish this border accurately because of the tissue distortion that follows posterior decortication. The boundary between the dorsal and ventral portions of the lateral geniculate nucleus was always easily distinguished, and all cells within the ventral lateral geniculate nucleus were normal. The pretectal area appeared normal in all cases.

Behavior. The preoperative acquisition of the brightness discrimination is presented in Figure 2. This figure shows, as individual bars, the mean errors to criterion for each of the three experimental groups. An analysis of variance indicated that there were no significant differences between the groups $(\mathrm{F}<1)$. A similar analysis computed on mean number of trials run during shaping and discrimination training also failed to reveal any differences between the groups $(F=1.56$, df $=2,14)$.

While the relationship between acquisition efficiency and incentive was not a major concern of the present research, it is important that the shock intensities we used did not produce wide differences in learning. This is so because different acquisition rates would, by definition, produce different amounts of experience with the apparatus and this could confound the purpose of our investigation. However, we wish to emphasize that these data should not be interpreted as indicating that incentive has little influence on acquisition rate. Rather, the present results simply suggest that the range of shock intensities of the present experiment was not sufficient to produce any significant differences in acquisition.

However, simply because each group required a similar number of trials and committed a similar number of errors attaining criterion, this does not mean that each group also received a similar amount of shock. Accordingly, we computed the average number of shock pulses per trial received by the animals of each of the three experimental groups during both shaping and formal discrimination training. These data are presented in Figure 3, which shows that, contrary to the number of trials or the number of errors, there were significant differences between the three groups of animals $(F=10.3$, $\mathrm{df}=2,14, \mathrm{p}<.01)$. Using the Duncan multiple range procedure, it was determined that the .3-mA group received a significantly greater number of shocks than either the .5-mA group or the .8-mA group, while these latter two groups did not differ

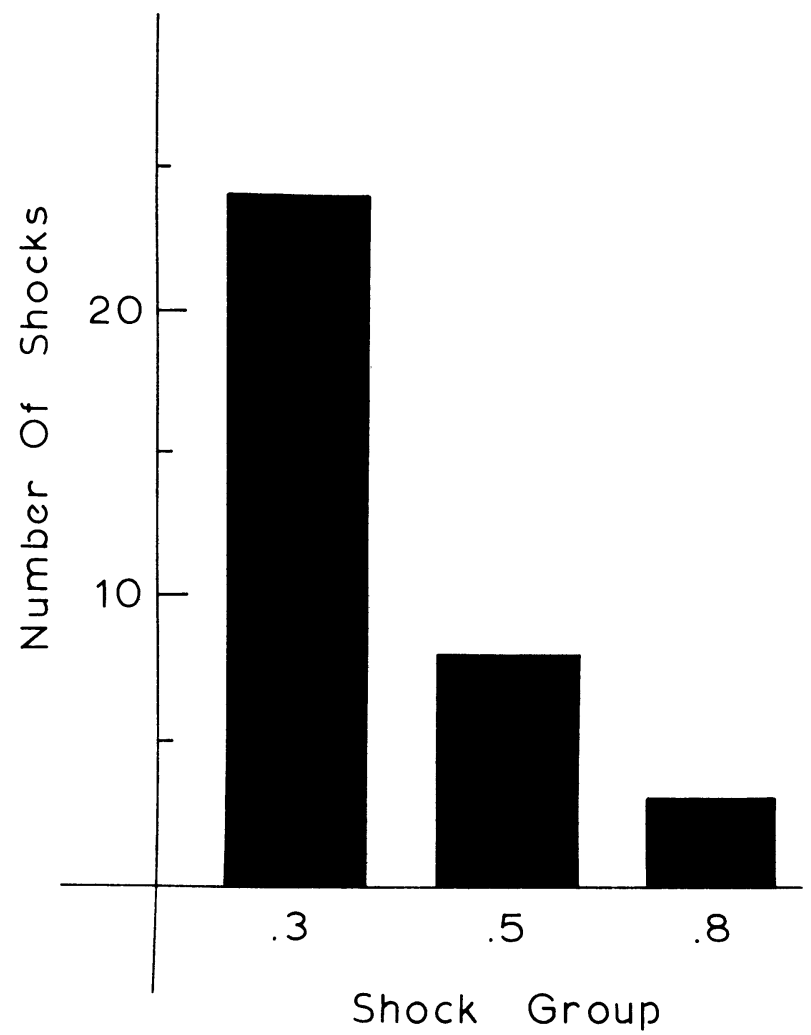

Figure 3. Mean number of shock pulses experienced by the animals of each experimental group during both preoperative shaping and discrimination training. 
significantly. Also, it is of some importance to note that an estimate of the total current experienced by the animals of each group (multiplying shock intensity times the number of shocks received) showed similar results. The respective figures for the .3-, $.5-$, and .8-mA shock groups are 664, 346, and $156 \mathrm{~mA}$ total current. An analysis of variance on these data was also significant $(\mathrm{F}=8.49, \mathrm{df}=2,14$, $\mathrm{p}<.01$ ), and the Duncan multiple range test again showed that the .3-mA group received a significantly greater amount of current than either the .5- or the .8-mA groups which were not significantly different from one another. Thus, even though there were no significant differences in the number of trials or number of errors to criterion performance, the .3-mA group nonetheless experienced both a greater number of shocks and a greater total current in attaining this criterion.

Turning to the postoperative performance of the three experimental groups, Figure 4 shows the mean response latency of each group during the 10-trial

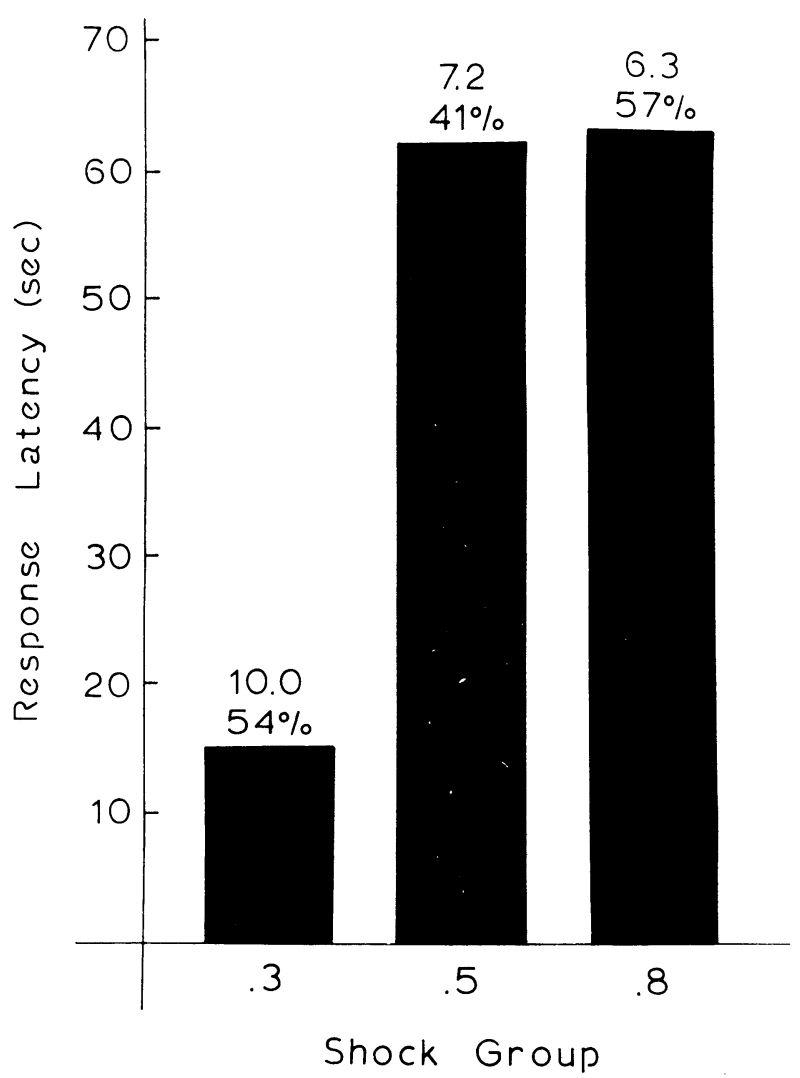

Figure 4. Mean response latency during the 10-trial postoperative test for the animals preoperatively trained with .3-, .5-, and .8-mA shock intensity. During this postoperative test, no shock was administered. The topmost number above each bar represents the number of test trials where the animal responded. The lower number above each bar represents the percentage of these responses where the animal chose the goalbox associated with the preoperatively correct cue. postoperative retention test. The topmost number above each individual bar is the mean number of times the animals of that particular group responded. The lower number is the percentage of those completed responses which were to the goal area signified by the preoperatively correct discriminative cue. Neither of these measures distinguished between the experimental groups $(F=2.06, \mathrm{df}=2,14$, for completed responses; and $F=.8$, df $=2,12$, for percentage of completed responses to the preoperatively correct cue). On the other hand, an analysis of variance showed that there were significant differences between the response latencies of the three experimental groups $(F=3.86$, df $=2,14, p<.05)$. A Duncan multiple range test indicated that the response latency of the .3-mA group was significantly shorter than the response latency of either the .5-mA group or the .8-mA group and that these latter two groups were not significantly different from one another.

On the basis of these data, we feel confident in concluding that the neural mechanisms related to the incentive to perform a particular behavior are, like the neural mechanisms associated with memory traces of the behavior, spared by visual decortication. And, while it is obviously of importance to understand the psychological and/or physiological basis of how incentive is related to particular behaviors and postoperative sparing, the point that is critical is that such sparing occurred, for whatever reason. That is, we simply wished to determine whether the disruption of a brightness discrimination following a visual neodecortication might be related to a dysfunction in the animal's motivation to perform the behavior. Since preoperative experience with different levels of incentive can differentially influence postoperative behavior, it would seem reasonable to conclude that the neural mechanisms associated with the motivation to perform are spared and that the disruption of a learned brightness discrimination is a function of something else.

\section{EXPERIMENT 2}

Given that it is possible to demonstrate that the memory traces of, and the motivation to perform, a learned brightness discrimination survive the visual decortication, one might justifiably inquire as to why there is a behavioral deficit at all. Part of the answer to this somewhat embarrassing question may be that the existence of particular neural mechanisms does not necessarily mean that these neural mechanisms will be utilized. Indeed, certain recent research has suggested that the behavioral dysfunction following neocortical injury may, in fact, represent a fundamental problem in utilization. For example, Yeterian, Waters and Wilson (1976) report that the normal rat 
is able to benefit from (utilize) what is learned with one sensory modality in the acquisition of similar behaviors involving a different sensory modality. However, when a striate lesioned rat acquires a brightness discrimination, this acquisition does not benefit the subsequent acquisition of an auditory intensity discrimination. Since retention is similar in the normal and destriate rat, the fact that a learned brightness discrimination will not benefit acquisition of an auditory discrimination in the brain-injured animal could indicate an inability on the part of the neocortically injured rat to utilize what is available.

The LeVere and Davis (1977) results previously noted with regard to Experiment 1 may be similarly interpreted as a dysfunction in utilization. In this research, it should be remembered that normal rats are fully able to utilize what is learned under one motivational state when acquiring discriminations under a different motivational state. However, if a visual decortication intervenes between the initial learning and the subsequent relearning, then the preoperatively learned brightness discrimination has little effect on postoperative behavior. Since the memory traces of the preoperatively acquired brightness discrimination are spared by visual decortication (LeVere \& Davis, 1977; LeVere \& Morlock, 1973, 1974), it would once again appear that the neocortically injured animal suffers a problem which might be characterized by an inability to utilize what is potentially available.

However, two considerations temper the unequivocal acceptance of the proposition that the behavioral dysfunction following neocortical injury may represent a utilization deficit. First, Yeterian et al. were concerned with intermodal utilization. Second, LeVere and Davis were concerned with postoperative utilization of behaviors established before brain injury. In both cases, the research involved something more than a simple question of utilization. Thus, before seriously proposing that the behavioral deficit following neocortical ablation involves a dysfunction in the utilization of available neural mechanisms, it must be demonstrated that this dysfunction is independent of other factors. That is, if the cortically lesioned rat suffers an inability to fully utilize spared neural mechanisms, then this inability should occur even when: (1) all testing is within the same sensory modality, and (2) all testing occurs subsequent to brain injury.

The final experiment is an attempt to demonstrate this and raises the question of whether previously lesioned rats will utilize what is learned under one motivational state in the performance of behaviors under a different motivational state. Specifically, using the reversal training paradigm, one would predict that if the behavioral dysfunction following visual decortication is an impairment in utilization, then a previously lesioned and trained rat should perform similarly when subsequently retrained on the original brightness discrimination or a reversal of the original brightness discrimination when motivation is changed.

\section{Method}

Subjects. The subjects for this experiment were 27 male hooded rats between the ages of 90 and 100 days at the time of surgery. All animals were maintained as described in Experiment 1, except that they were $23 \mathrm{~h}$ water deprived when trained for water reinforcement.

Apparatus. The apparatus was the same as that used in Experiment 1 . However, during water-reinforced training, a small quantity of water was placed in each goal compartment. But, since the entrance to the incorrect goal compartment was locked, the animal could obtain reinforcement only in the correct goal compartment. As with shock avoidance training, a correction procedure was used to allow the animal to obtain reinforcement on each and every trial.

Procedure. Two weeks prior to discrimination training, the visual neocortex of each animal was removed by aspiration, as described for Experiment 1. During the last 3 days of the 2-week postoperative rest period and during all water-reinforced discrimination training, the animals were $23 \mathrm{~h}$ water deprived. On Postoperative Days 15-17, the animals were shaped to run on the Yerkes apparatus to obtain water reinforcement. Following this, the animals were trained on the brightness discrimination at a rate of 10 trials per day. Fifteen of the animals were trained with the brighter (64-fL) cue as the correct cue, while the remaining 12 were trained with the dimmer (1-fL) cue as the correct cue. Training was continued until each animal attained a criterion of 9 first-choice correct responses within a day's training.

After criterion, all animals were allowed free access to food and water and given an additional 2-week rest. Subsequently, the animals were returned to the Yerkes apparatus and retrained on the previously learned brightness discrimination. However, during this training each animal was satiated and each responded to avoid/ escape footshock. Nine of the animals originally trained with the bright cue as the correct cue and five of the animals originally trained with the dim cue as the correct cue were retrained on the same discrimination as previously learned for water reinforcement. The other animals of these two groups were retrained with the reward contingencies of the previously learned brightness discrimination reversed. With the exception of the change in incentive and the fact that some animals were retrained on the reversal of the original brightness discrimination, all aspects of the second training session were identical to those of the initial training session. Our prediction was that if neocortical injury interfered with the utilization of neural mechanisms established during the initial training, then there should be no difference between the animals now retrained on the original brightness discrimination and the animals retrained on the reversal of the original brightness discrimination.

After retraining to criterion on the second brightness discrimination, each animal was sacrificed and treated as described in Experiment 1 .

\section{Results and Discussion}

Histology. The extent of the neocortical injury and amount of retrograde degeneration within the dorsal lateral geniculate nucleus is shown in Figure 5. The upper portion of the figure represents the animal sustaining the smallest lesion (45\% of neocortex), while the lower portion of the figure represents the animal sustaining the largest lesion (58\% of neo- 

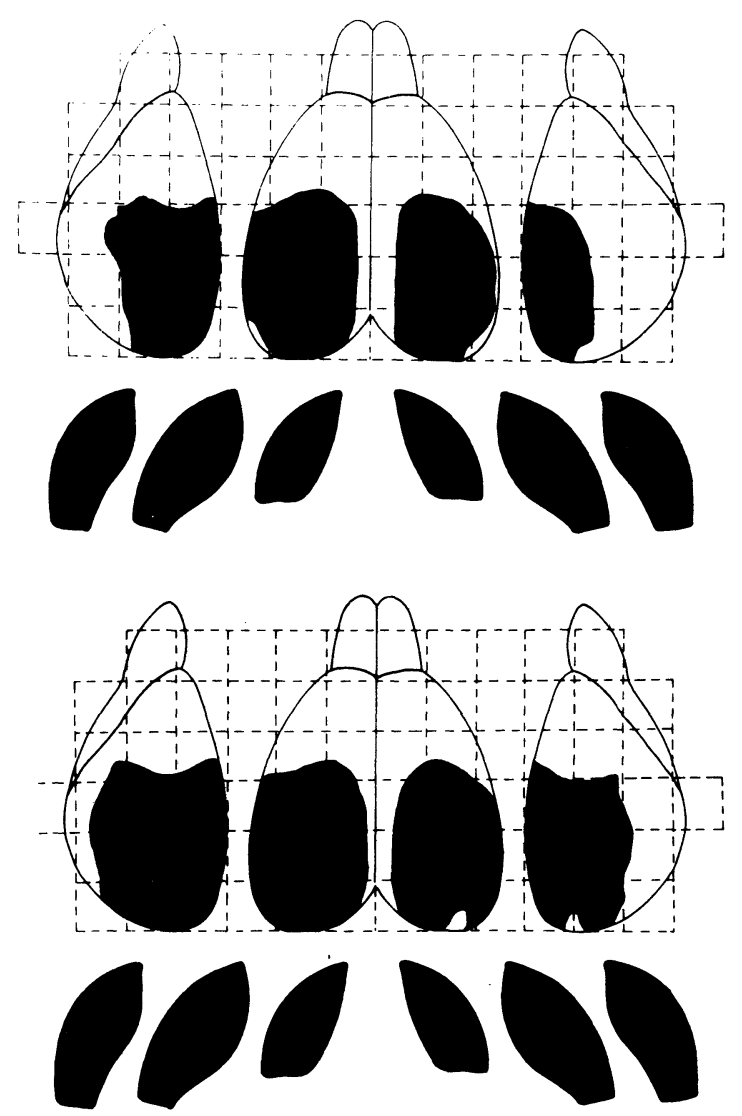

Figure 5. Neocortical surface and dorsal lateral geniculate nucleus diagrams for the rat sustaining the smallest amount of neocortical destruction (top) and the rat sustaining the largest amount of neocortical destruction (bottom). Neocortical destruction and retrograde degeneration within the dorsal lateral geniculate nucleus are indicated by blackened areas. The three frontal sections through the dorsal lateral geniculate nucleus correspond to approximately $2.5 \mathrm{~mm}$ (extreme left ard right), $3.1 \mathrm{~mm}$ (second from left and right), and $3.7 \mathrm{~mm}$ (middle) from the interaural line.

cortex). Grouping the animals on the basis of (1) the brightness of the correct cue during initial training, (2) the brightness of the correct cue during retraining, or (3) whether the animals were retrained on the original brightness discrimination or a reversal of the original brightness discrimination, showed no significant differences in lesion size ( $t$ test, independent samples).

Examination of the cresyl-violet-stained sections gave results identical to those described for Experiment 1 .

Behavior. The behavioral data are presented in Figure 6, which shows mean errors to criterion during the initial training session and during the second retraining session. The curves group the animals according to whether they were retrained on the originally learned brightness discrimination or a reversal of the originally learned brightness discrimination. The performance of the lesioned animals of the pres- ent experiment is presented in the top set of curves. As can be seen from these curves, the initial learning for water reinforcement for both the nonreversal and the reversal animals is identical. This is, of course, expected, since the animals were deliberately matched. However, during the second retraining session to avoid footshock, the animals of the reversal group were quite impaired. Apparently, visual neodecortication does not prevent the animal from attempting to utilize what was learned under one motivational condition during acquisition and performance of a similar behavior under a different motivational condition. In fact, comparing the performance of the striate-lesioned animals with identically treated normal animals (LeVere \& Davis, 1977, Experiment I) indicates that the lesioned rat is quite similar to the normal except for a general performance deficit which we and others have previously reported (Cooper, Freeman, \& Pinel, 1967; Lashley, 1930; LeVere \& Mills, 1977).

Statistically, the error scores presented in Figure 6 were analyzed with a 2 by 2 factorial analysis of variance. The two main factors were: (1) brightness of the correct cue during the second training session, and (2) whether or not the animals were retrained on the originally learned brightness discrimination or on a reversal of the originally learned brightness discrimination. Both of these main effects were significant $(\mathrm{F}=9.39$, $\mathrm{df}=1,23$ for the brightness main effect; $F=9.53$, df $=1,23$ for the type of problem main effect). The interaction between these two main effects was not significant $(F<1)$. This result exactly

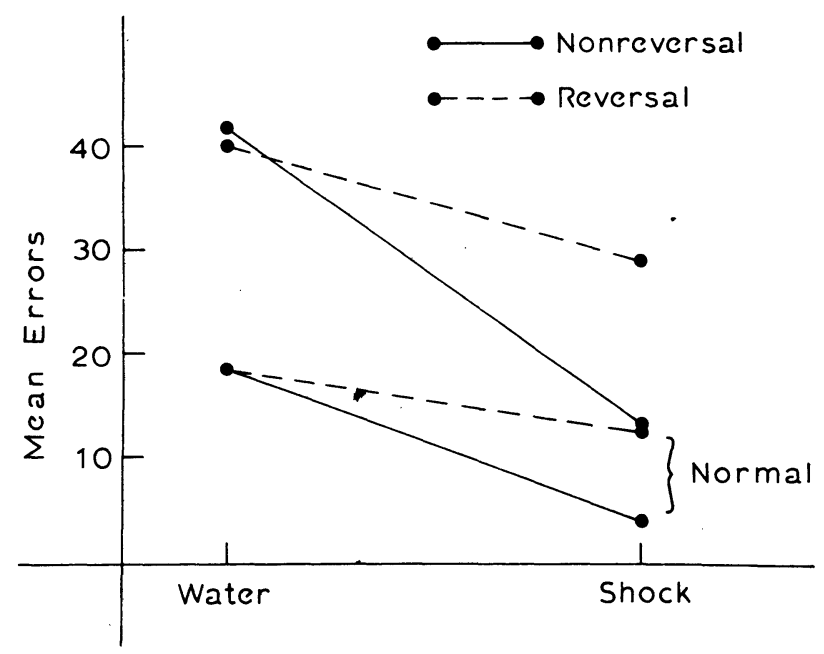

Figure 6. Mean errors to criterion for those animals retrained on the initially learned brightness discrimination (nonreversal) and those animals retrained on the reversal of the initially learned brightness discrimination (reversal). The top two sets of curves present the data from the striate lesioned rats of the present experiment while the bottom two sets of curves are data from normal animals of our previous research (LeVere \& Davis, 1977, Experiment I). 
replicates what was previously found with normal animals (LeVere \& Davis, 1977), and validates the graphic similarity between the normal and the striate rats shown in Figure 6. Thus, whatever the striate rat's problem is, it is not a general inability to utilize previously established memory traces or neural mechanisms.

\section{GENERAL DISCUSSION}

With appropriate methodology, it is possible to demonstrate that the inability to initially perform a learned brightness discrimination following visual neodecortication is not due to the destruction of the neural mechanisms underwriting the memory traces of the behavior (LeVere \& Davis, 1977; LeVere \& Morlock, 1973, 1974). The present research extends these results by demonstrating that this inability is also not due to either (1) destruction of the neural mechanisms associated with the motivation to perform the behavior (Experiment 1), or (2) the destruction of the neural mechanisms which allow utilization of previously acquired behaviors (Experiment 2). In both cases, the lesioned rat's behavior was not independent of previous experience. And in both cases, one must necessarily conclude that the neural mechanisms of that particular aspect of the behavior were spared and able to function subsequent to the visual decortication. Given this sparing of not only the neural traces of a preoperatively learned behavior, but also the motivation to perform the behavior and the ability to utilize previously learned behaviors, the principal question again centers on why there is a behavioral deficit at all.

As a possible, and perhaps elementary, explanation of the behavioral deficit, we may start by noting the differential consequences of changing motivation between original learning and subsequent relearning when (1) the rat suffers the brain insult between the two training situations, and (2) the rat suffers the neural insult prior to either training situation. In the first case, we have previously shown (LeVere \& Davis, 1977) that changing the motivation between preoperative original learning and postoperative retraining yields comparable performances between animals postoperatively retrained on the originally learned brightness discrimination and animals postoperatively retrained on the reversal of the original brightness discrimination. Since there is no reversal deficit, it would seem reasonable to conclude that the memory traces that are spared by the visual decortication do not influence postoperative behavior. However, as demonstrated by the present experiments, if the lesion occurs prior to initial learning, then what is learned under one motivational state will strongly influence subsequent learning under a different motivational state. The critical issue, then, is the temporal relationship between the lesion and the initial learning and later relearning (recovery) situations and why this differentially affects availability and/or utilization.

At first glance, one might attempt to explain these differential effects on the grounds that a neocortical lesion will induce structural or functional reorganization of neural mechanisms. The occurrence of either of these could obviously change the availability (utilization) of preoperatively established neural traces and affect postoperative utilization. However, this would not necessarily be the case for neural traces acquired subsequent to brain injury, because these would involve the reorganized neural mechanisms and thus utilization would not suffer. The difficulty with this posture is that it does not square well with the fact that if preoperative and postoperative motivation are the same, then preoperatively established neural traces are available and utilized by the lesioned animal. In other words, if the nervous system undergoes some sort of reorganization in response to damage, then why does this adversely affect the availability and utilization of neural mechanisms only when motivation is changed? The notion of vicariation, of course, suffers a similar inconsistency and thus fares little better than reorganization as an explanation of the present results.

Given the inadequacy of these somewhat more exotic postulations of the effects of brain injury, we suggest that a more viable alternative might rest on traditional behavior theory and a single, not terribly unreasonable, assumption. The assumption is that both changes in motivation and losses of primary sensory neocortex have perceptual consequences. In the case of motivation, these consequences involve the individual's perception of its intrinsic state, while in the case of sensory neocortical ablation, these consequences involve the individual's perception of the extrinsic environment. For example, a change in motivation can serve as a discriminative cue (Hull, 1933; Kendler, Karasik, \& Schrier, 1954; Kendler \& Levin, 1951; Kendler, Levin, Altchek, \& Peters, 1952; Leeper, 1935; Wickens, Hall, \& Reid, 1949). To serve as a discriminative cue, changes in motivation must, by definition, be perceptually distinct. Considering the perceptual effects of sensory neocortical ablation, Lavond, Hata, Gray, Geckler, Meyer, and Meyer (1978) have shown that while normal rats can learn to discriminate oblique line patterns, visual decorticate rats cannot. However, it is well established that the striate rat is fully capable of learning a brightness discrimination, so the pattern discrimination deficit must not be a learning deficit but, rather, an inability to detect oblique line patterns, i.e., a perceptual effect.

Accepting that both changes in motivation and sensory cortex lesions have perceptual consequences, 
we may then view the present data from the vantage point of the traditional concept of transfer of training. Within this framework, and following Osgood's (1949) eloquent analysis, whether a particular behavior will influence a subsequent behavior is principally determined by the similarity of the intrinsic and extrinsic conditions over which this influence must extend. Since perceptual changes obviously decrease the similarity between two situations, a change in motivation or a sensory cortex ablation will mitigate transfer of training. And, since there is no particular reason to believe that the perceptual consequences of a change in motivation and the perceptual consequences of sensory cortex ablation are not cumulative, it is fairly easy to appreciate the present set of data. For example, the perceptual effects of either a change in motivation or a sensory cortex ablation alone are probably not sufficient to eliminate transfer of training. Thus, preoperatively acquired memory engrams can and do influence postoperative behavior, provided nothing else serves to decrease the similarity between the preoperative and postoperative situations. Similarly, acquisition under one motivational state will influence subsequent performance under a different motivational state, provided nothing else serves to decrease the similarity between the two situations. However, the combined perceptual effects of a sensory neocortex ablation and a change in motivation might well produce such a degree of dissimilarity that transfer would not occur, with the result that spared neural traces would not influence the animal's postoperative behavior. Or, from the animal's point of view, it is not a question of utilizing what is available but, rather, a question of applying what is compatible.

To support this explanation, we present the data shown in Figure 7. Estimating from the figures of Mackintosh, McGonigle, Holgate, and Vanderver (1968), there is approximately a 20-error impairment when normal animals are retrained on the reversal of a previously learned brightness discrimination. This reversal impairment is, however, lessened to 9 errors if the normal rat's motivation changes between the initial training and the subsequent retraining (LeVere \& Davis, 1977). Similarly, there is only a 9-error reversal impairment if motivation is unchanged but the rat suffers striate decortication between the initial training and the subsequent retraining (LeVere $\&$ Morlock, 1973). And finally, the reversal impairment is but 12 errors if a lesioned rat is initially trained under one motivational state and then retrained under a different motivational state (Experiment 2). In other words, a motivational change between two training situations in the normal animal, a motivational change between two training situations in the lesioned animal, or a sensory cortex ablation between two training situations will roughly have the normal

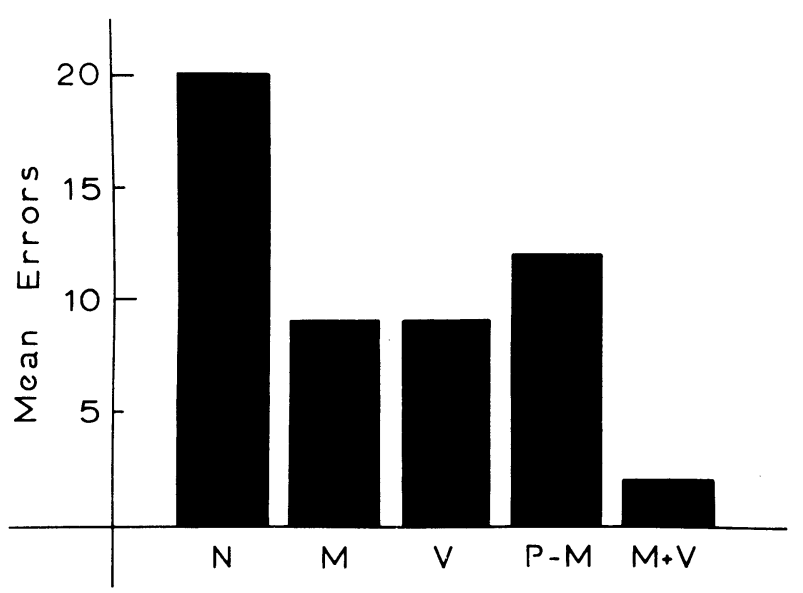

Figure 7. The reversal deficit expressed in mean errors to criterion during reversal training when: (1) normal animals are retrained without any change other than the reversal (N); (2) normal animals are retrained on the reversal problem under a different motivational state (M); (3) animals are retrained on the reversal problem subsequent to a visual decortication (V); (4) lesioned animals are trained and then retrained on the reversal problem under a different motivational state (P-M); (5) animals are retrained on the reversal problem subsequent to both a change in motivational state and a visual decortication $(M+V)$.

reversal impairment. And, for the same basic reason, in each case the manipulation causes a perceptual change which decreases the similarity between the two situations, producing less transfer and less prograde interference. On the other hand, if the rat must endure both a change in motivation and a sensory cortex ablation, then the combined perceptual consequences of these events are sufficient not to lessen, but to prevent transfer, and thus there is no prograde interference, and thus the typical reversal impairment disappears (LeVere \& Davis, 1977).

It is on these grounds that we argue that the nature of the behavioral deficit following neocortical insult is not a loss of neural mechanisms underlying memory engrams, is not a loss of the neural mechanisms underlying the motivation to perform the behavior, and is not a loss of neural mechanisms underlying the utilization of established behaviors. We also argue that the differential effectiveness of spared preoperative neural mechanisms is not related to any unique response to brain injury such as reorganization or vicariation. Rather, it is our position that the general effects of brain injury and the particular results of the present set of experiments are more easily appreciated under the rubric of classic behavior theory, i.e., transfer of training. Moreover, until firm contradictory evidence presents itself, we would suggest that recovery of function may be most profitably considered as an optimizing process representing the lesioned animal's maximization of whatever normal capacities of whatever neural mechanisms remain subsequent to the brain insult. 


\section{REFERENCES}

Akert, K., Orth, O. S., Harlow, H. F., \& Schlitz, F. Learned behavior of rhesus monkeys following neonatal bilateral prefrontal lobotomy. Science, 1960, 132, 1944-1945.

Benjamin, R. M., \& Thompson, R. F. Differential effects of cortical lesions in infant and adult cats on roughness discrimination. Experimental Neurology, 1959, 1, 305-321.

Chow, K. L., \& Survis, J. Retention of overlearned visual habit after temporal cortical ablation in monkey. Archives of Neurology and Psychiatry, 1958, 79, 640-646.

Cooper, R. M., Freeman, I., \& Pinel, J. P. J. Absolute threshold of vision in the rat after removal of striate cortex. Journal of Comparative and Physiological Psychology, 1967, 64, 36-39.

Dawson, R. G., Conrad, L., \& Lynch, G. Single and twostage hippocampal lesions: A similar syndrome. Experimental Neurology, 1973, 40, 263-277.

Finger, S. Lesion momentum and behavior. In S. Finger (Ed.), Recovery from brain damage. New York: Plenum, 1978.

Glendenning, R. L. Effects of training between two unilateral lesions of visual cortex upon ultimate retention of black-white discrimination habits by rats. Journal of Comparative and Physiological Psychology, 1972, 80, 216-229.

Hughes, H. C. Anatomical and neurobehavioral investigations concerning the thalamo-cortical organization of the rat's visual system. Journal of Comparative Neurology, 1977, 175, 311-335.

Hull, C. L. Differential habituation to internal stimuli in the albino rat. Comparative Psychology, 1933, 16, 255-263.

IsAACSON, R. L. The myth of recovery from early brain damage. In N. R. Ellis (Ed.), Aberrant development in infancy. Hillsdale, N.J: Erlbaum, 1975.

IsaAcson, R. L., \& Schmaltz, L. W. Failure to find savings from spaced, two-stage destruction of hippocampus. Communications in Behavioral Biology, 1968, 1, 353-359.

Johnson, D., \& Almli, C. R. Age, brain damage, and performance. In S. Finger (Ed.), Recovery from brain damage. New York: Plenum, 1978.

Kendler, H. H., \& Levin, S. Studies of the effect of drive: I. From hunger tc thirst in a T-maze. Journal of Experimental Psychology, 1951, 41, 429-436.

Kendler, H. H., Levin, S., Altchek, E., \& Peters, H. Studies of the effect of change of drive: II. From hunger to different intensities of a thirst drive in a T-maze. Journal of Experimental Psychology, 1952, 44, 1-3.

Kendler, H. H., Karasik, A. D., \& Schrier, A. M. Studies of the effect of drive: III. Amounts of switching produced by shifting drive from thirst to hunger and from hunger to thirst. Journal of Experimental Psychology, 1954, 47, 179-182.

KenNaRD, M. A. Age and other factors in motor recovery from precentral lesions in monkeys. American Journal of Physiology, 1936, 115, 138-146.

Kennard, M. A. Reorganization of motor function in the cerebral cortex of monkeys deprived of motor and premotor areas in infancy. Journal of Neurophysiology, 1938, 1, 477-497.

KenNard, M. A. Cortical reorganization of motor function. Studies on series of monkeys of various ages from infancy to maturity. Archives of Neurology and Psychiatry, 1942, 48, 227-240.

Kircher, K. A., Braun, J. J., Meyer, D. R., \& Meyer, P. M. Equivalence of simultaneous and successive neocortical ablations in production of impairments and retention of black-white habits in rats. Journal of Comparative and Physiological Psychology, 1970, 71, 420-425.

LASHLEY, K. S. Studies of cerebral function in learning: II. The effects of long-continued practice upon cerebral localization. Journal of Comparative Psychology, 1921, 1, 453-468.

LASHLEY, K. S. The mechanisms of vision: II. The influence of cerebral lesions upon the threshold of discrimination for bright- ness in the rat. Journal of Genetic Psychology, 1930, 37, 461-480.

Lavond, D., Hata, M. G., Gray, T. S., Geckler, C. L., Meyer, P. M., \& Meyer, D. R. Visual form perception is a function of the visual cortex. Physiological Psychology, 1978, $6,471-477$.

LEEPER, R. The role of motivation in learning: A study of the phenomenon of differential motivational control of the utilization of habits. Journal of Genetic Psychology, 1935, 46, 3-40.

LEVERE, T. E. Recovery of function after brainstem lesions in the rat. Journal of Comparative and Physiological Psychology, 1969, 69, 339-344.

LEVERE, T. E., \& DAvis, N. Recovery of function after brain damage: The motivational specificity of spared neural traces. Experimental Neurology, 1977, 57, 883-899.

LeVere, T. E., \& Mills, J. Residual differential brightness thresholds following removal of visual neocortex in rats. Physiological Psychology, 1977, 5, 490-496.

LeVere, T. E., \& Morlock, G. W. The nature of visual recovery following posterior decortication in the hooded rat. Journal of Comparative and Physiological Psychology, 1973, 83, 62-67.

LeVere, T. E., \& Morlock, G. W. The influence of preoperative learning on the recovery of a successive brightness discrimination following posterior neodecortication in the hooded rat. Bulletin of the Psychonomic Society, 1974, 4, 507-509.

LEVERE, T. E., \& WE ISS, J. Failure of seriatim dorsal hippocampal lesions to spare spatial reversal behavior in rats. Journal of Comparative and Physiological Psychology, 1973, 82, 205-210.

Lukaszewska, I., \& Thompson, R. Retention of an overtrained pattern discrimination following pretectal lesions in rats. Psychonomic Science, 1967, 8, 121-122.

Mackintosh, N. J., McGonigle, B., Holgate, V., \& VANDERVER, V. Factors underlying improvement in serial reversal learning. Canadian Journal of Psychology, 1968, 22, 85-95.

Meyer, D. R., Hughes, H. C., Buchholz, D. J., Dalhouse, A. D., Enloe, L. J., \& Meyer, P. M. Effects of successive unilateral ablations of principalis cortex upon performance of delayed alternation and delayed response by monkeys. Brain Research, 1976, 108, 397-412.

Orbach, J., \& Fantz, R. L. Differential effects of temporal neocortical resections on overtrained and nonovertrained visual habits in monkeys. Journal of Comparative and Physiological Psychology, 1958, 51, 126-129.

Osgood, C. E. The similarity paradox in human learning: A resolution. Psychological Review, 1949, 56, 132-143.

RAAB, D. H., \& AdES, H. W. Cortical and midbrain mediation of a conditioned discrimination of acoustic intensities. American Journal of Psychology, 1946, 59, 59-83.

Thatcher, R. W., \& Kimble, D. P. Affects of amygdaloid lesions on retention of an avoidance response in overtrained and nonovertrained rats. Psychonomic Science, 1966, 6, 9-10.

Weese, G. D., Neimand, D., \& Finger, S. Cortical lesions and somesthesis in rats: Effects of training and overtraining prior to surgery. Experimental Brain Research, 1973, 16, 542-550.

Wickens, D. D., Hall, J., \& Reid, L. S. Associative and retroactive inhibition as a function of the drive stimulus. Journal of Comparative and Physiological Psychology, 1949, 42, 398-403.

Yeterian, E. H., Waters, R. S., \& Wilson, W. A. Posterior cortical lesions and specific crossmodal transfer in the rat. Physiological Psychology, 1976, 4, 281-284.

(Received for publication December 12, 1978; revision accepted October $28,1979$. ) 